\title{
New Magnetic Circuit Structure of Hall Current Sensor for Reducing Conductor Position Error
}

\author{
Chao Tan, Chenguang Wu, Xiaohui Gong, Xinglin Li, Ruijie Fu, and Zhoumei Yue \\ College of Electrical Engineering and New Energy, China Three Gorges University, \\ 8 University Road, Yichang, 443000, China
}

(Received April 22, 2020; accepted July 15, 2020)

Keywords: current measurement, finite element, Hall current sensor, magnetic induction intensity, position error

To reduce the error of a Hall current sensor caused by the variation of the conductor position, a new symmetrical dual-core four-air-gap magnetic circuit structure is designed. The proposed structure includes two symmetrically placed dual-air-gap magnetic cores and four Hall devices, where the two symmetrically placed dual-air-gap magnetic cores are used as the sensor magnetic circuit and the average voltage output from the four Hall devices is used as the output of the sensor. First, the output error of a traditional Hall current sensor caused by the variation of the conductor position is discussed, and the principle of reducing the error by using a symmetrical dual-core four-air-gap magnetic circuit structure is analyzed. Then, a finite element simulation was performed to mimic the magnetic induction intensity at the air gap of a single-core single-air-gap magnetic core and that of the proposed circuit structure. Then, the relative error of these two circuits was calculated. Finally, a prototype of the proposed circuit structure was manufactured. The simulation and experimental results show that the proposed circuit structure can significantly reduce the measurement error caused by the position deviation of the measured conductor, and the maximum measurement error is only $0.566 \%$ when the measured current ranges from 10 to $50 \mathrm{~A}$.

\section{Introduction}

Considerable changes have taken place in the field of electric vehicles with the development of technology. Electric vehicles with zero-emission characteristics have been developed by different countries around the world, and an increasing number of governments are encouraging research on electric vehicles. The charging pile is a device that charges the battery of electric vehicles. The measurement accuracy of a current sensor is important for accurately measuring charging capacity and current. The measurement accuracy of an AC energy meter installed on an AC charging pile should be better than $0.5 \%$ in accordance with an international standard. ${ }^{(1)}$ Therefore, it is important to develop high-precision, low-cost current sensors for electric energy measurement.

*Corresponding author: e-mail: ctgutc@ctgu.edu.cn

https://doi.org/10.18494/SAM.2020.2902 
Current sensors can be classified into contact and contactless types according to the measurement method. The contactless type has obvious advantages and has been extensively studied in recent years. Contactless current sensors include the fluxgate current sensor, Rogowski coil sensor, current transformer, and Hall current sensor. ${ }^{(2-6)}$ Hall current sensors are suitable for use with charging piles owing to their high sensitivity, low cost, low power consumption, and noncontract measurement. The current can be obtained indirectly by measuring the magnetic field around the energized conductor. At present, there are two types of Hall current sensors: sensors with and without a magnetic flux concentrator. ${ }^{(7-15)}$ To improve the accuracy of the measuring system and to reduce the crosstalk effects of other magnetic fields, a circular array of magnetic sensors without a magnetic concentrator was proposed. ${ }^{(7)}$ The sensitivity of a Hall current sensor with a concentrator can be markedly improved because of the magnet congregate effect, and the influence of external magnetic fields can also be considerably reduced. Magnetic concentrator structures are classified into openloop and closed-loop Hall current sensors. Popovic et al. ${ }^{(10)}$ proposed a Hall current sensor with a magnetic concentrator based on integrated circuit technology and integrated packaging technology. The equivalent magnetic noise and offset of this Hall current sensor can be reduced by integrating magnetic flux concentrators on the sensor chip. To achieve a zero-flux condition and improve the performance of the sensor, a closed-loop Hall current sensor with a compensation coil was proposed. A closed-loop structure was designed by comprehensive research on the Hall element, air gap width, ferromagnetic material, and structure, in which the linearity and accuracy of the sensor reached 0.1 and $0.35 \%$ full-scale (FS), respectively, when the measured current ranged from 0 to $50 \mathrm{~A} .^{(15)}$

However, it is necessary to place the measured conductor at the center of the magnetic ring when a Hall current sensor with a magnetic concentrator is adopted. ${ }^{(5-17)}$ The common installation method is to fill the conductor into the magnetic ring aperture. This approach usually requires strict size limiting. If the conductor cannot fill the ring aperture, it is necessary to keep the conductor at the center of the ring. However, owing to the vibration and other reasons, the anchor points of the Hall current sensor and the wire are loosened, causing the conductor to move. When the measured conductor is moved, the output signal of the sensor has a large error, reducing measurement accuracy. As an important core component in automotive battery management system and controller, the Hall current sensor plays an important role. However, when driving an electric car, it is difficult to avoid the vibration of the electric car. Therefore, we propose a symmetrical dual-core four-air-gap magnetic core sensor structure to reduce the influence of the variation of the measured conductor position.

\section{Proposed Hall Current Sensor}

\subsection{Sensor structure}

As shown in Fig. 1(a), a magnetic core sensor with a symmetrical dual-core four-air-gap structure is adopted. Four Hall chips with identical characteristics are placed in the four air gaps of the dual cores. As shown in Fig. 1(b), owing to the magnet congregate effect of the 


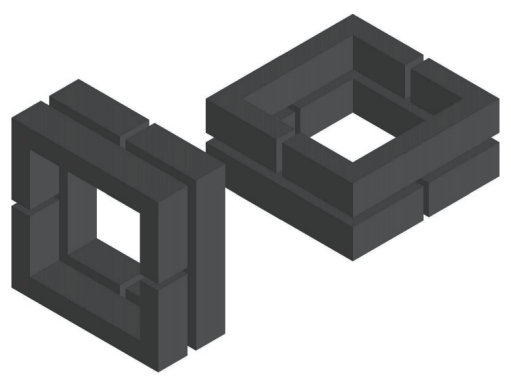

(a)

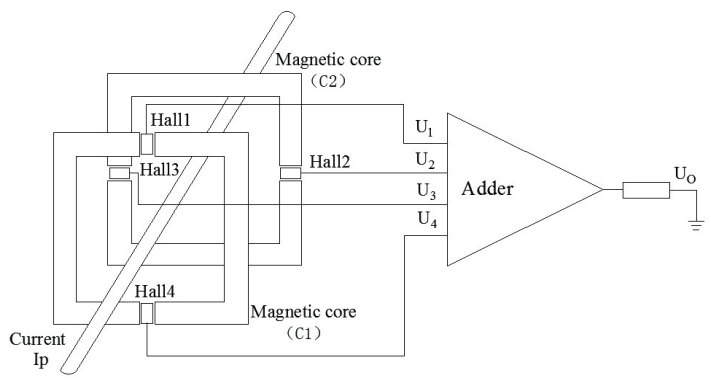

(b)

Fig. 1. (a) Symmetrical dual-core four-air-gap structure and (b) schematic diagram of proposed sensor.

magnetic core, the magnetic induction intensity at the four air gaps is proportional to the measured current $I_{P}$. Each of the four Hall chips will generate an output voltage. Then, four voltage signals are connected to an adder circuit composed of operational amplifiers. The position variation of the measured conductor will change the magnetic induction intensity in the air gap, resulting in the generation of different Hall voltages. To reduce the influence of position variation, four voltage signals are averaged, and the feedback resistance is adjusted to obtain the following output voltage $U_{o}$ of the sensor:

$$
U_{o}=\frac{1}{4}\left(U_{1}+U_{2}+U_{3}+U_{4}\right)
$$

where $U_{1}, U_{2}, U_{3}$, and $U_{4}$ are the voltage signals generated by the four Hall chips.

The above averaging operation can reduce the position error if more Hall devices are used in the magnetic ring. However, the magnet congregate effect of the magnetic ring is markedly affected by the width of the air gap. In practical applications, the magnetic ring has too many holes, and thus the magnet congregate effect will be greatly reduced, which will reduce the sensitivity of the sensor.

The models of single-core single-air-gap, dual-air-gap, and four-air-gap structures are established by finite element simulation using COMSOL software, in which the wires are located at the center of the magnetic ring, and the current is $10 \mathrm{~A}$. The selected core parameters are shown in Table 1. The simulation results are shown in Table 2. It can be seen from the single-core four-air-gap structure that the magnetic flux density of the whole magnetic ring is small. Therefore, considering the practical application and measurement requirements, it is reasonable to adopt the symmetrical dual-core four-air-gap structure.

\subsection{Error analysis}

For an ideal magnetic core model, the air gap is much smaller than the core. An infinitely long conductor is placed at the center of the core. According to Ampere's law, we have 
Table 1

Simulation core parameters.

\begin{tabular}{lcccc}
\hline Material & Outer side length $\left(\mathrm{mm}^{2}\right)$ & Inner side length $\left(\mathrm{mm}^{2}\right)$ & Height $(\mathrm{mm})$ & Size of air gap $(\mathrm{mm})$ \\
\hline Ferrite & $30 \times 30$ & $18 \times 18$ & 6 & 1.5 \\
\hline
\end{tabular}

Table 2

(Color online) Magnetic induction intensity with different numbers of air gaps.

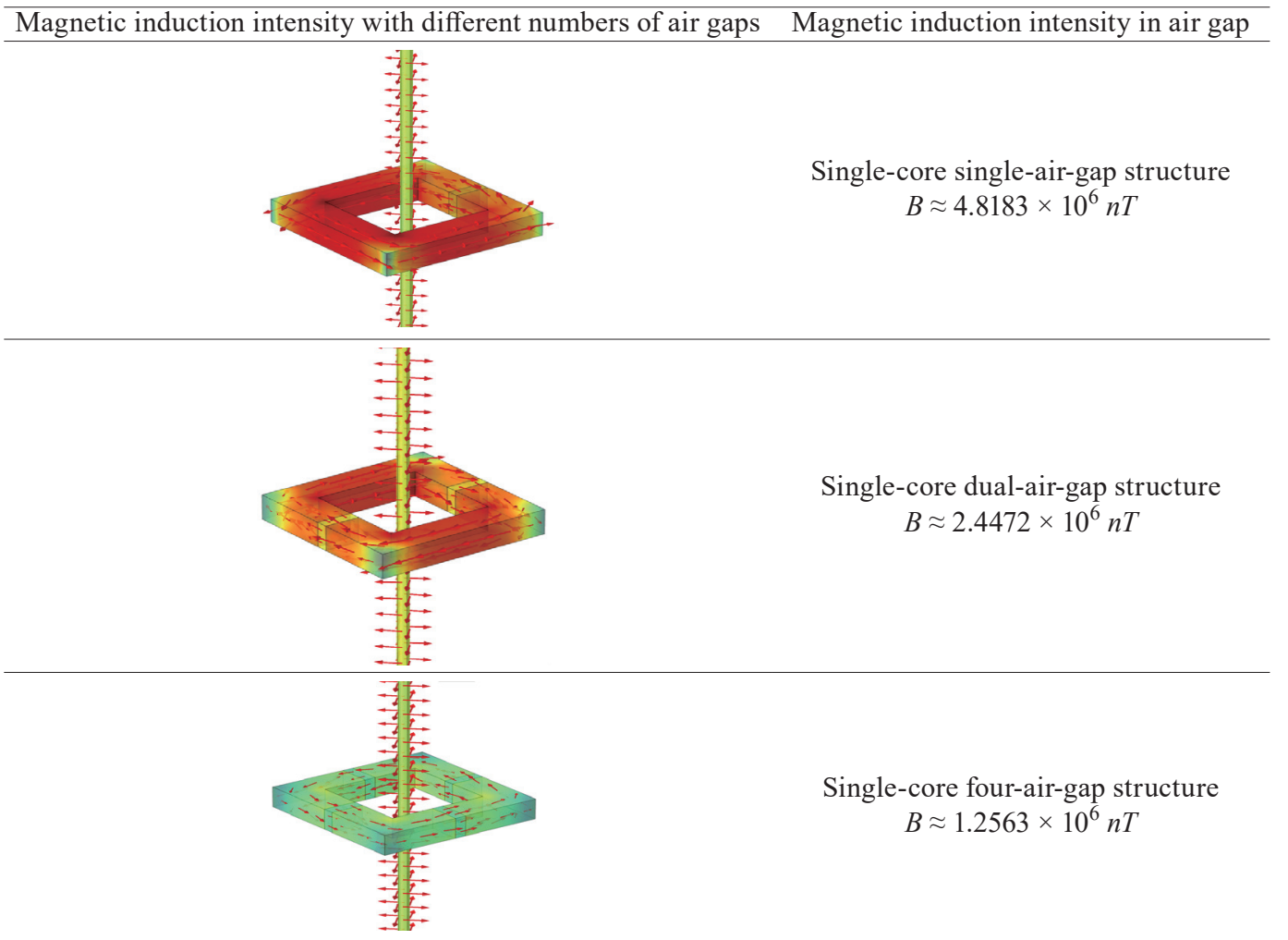

$$
N I_{P}=H_{c} l_{c}+H_{g} l_{g}
$$

where $H$ is the magnetic field strength, $I_{P}$ is the primary current, $l$ is the path length, and $N$ is the number of turns of the primary coil $(N=1$ in our case). The subscript $c$ refers to the core and the subscript $g$ to the air gap. Substituting $\mu=B / H$ into Eq. (2) gives

$$
I_{P}=\frac{B_{c} l_{c}}{\mu_{c}}+\frac{B_{g} l_{g}}{\mu_{g}},
$$

where $B$ is the magnetic induction intensity and $\mu$ is permeability. Assuming that the magnetic field at the air gap is continuous and uniform, by substituting $B_{c}=B_{g}$ into Eq. (3), we can obtain the magnetic induction intensity at the air gap: 


$$
B_{g}=\mu_{g} \mu_{c} \frac{I_{P}}{\mu_{g}\left(\pi \frac{D+d}{2}-l_{g}\right)+\mu_{c} l_{g}},
$$

where $D$ is the outer diameter of the magnetic ring and $d$ is the inner diameter of the magnetic ring. Because the magnetic permeability of the selected magnetic ring is extremely high, we have

$$
\mu_{c} l_{g} \gg \mu_{g}\left(\pi \frac{D+d}{2}-l_{g}\right) .
$$

Therefore, Eq. (5) can be simplified to

$$
B_{g}=\mu_{g} \frac{I_{P}}{l_{g}} .
$$

The magnetic induction intensity of the air gap is linearly related to the measured current $I_{P}$ according to the above analysis. Figure 2 shows the theoretical calculation results of the variation of the air-gap magnetic induction intensity with the measured current $I_{P}$ when different air gap widths are adopted. The smaller the air gap width, the more obvious the magnet congregate effect. Thus, the theoretical calculation results are consistent with the simulation results.

In addition, it can be seen from Eq. (4) that the magnetic induction intensity of the air gap will not change if the primary current is constant. However, there is magnetic flux leakage in the air gap. The magnetic induction intensity in the air gap will fluctuate, which is caused by the position variation of the conductor. Also, it is difficult to accurately calculate the magnetic flux leakage.

The COMSOL finite element software was used to simulate a single-core single-air-gap structure. The coordinate system is shown in Fig. 3, and the air gap relative coordinate is

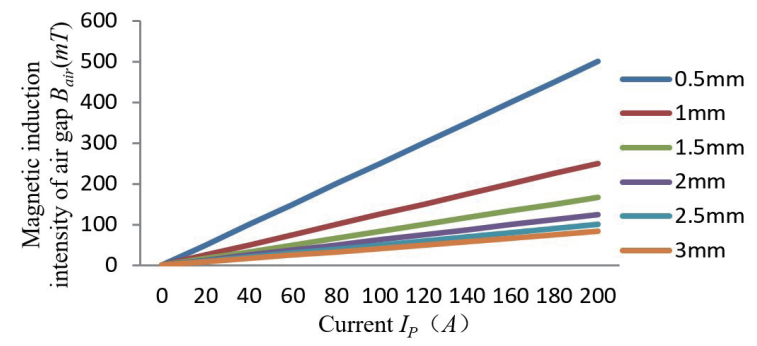

Fig. 2. (Color online) Theoretical calculations of magnetic induction intensity of air gaps of different widths as functions of the measured current $I_{P}$.

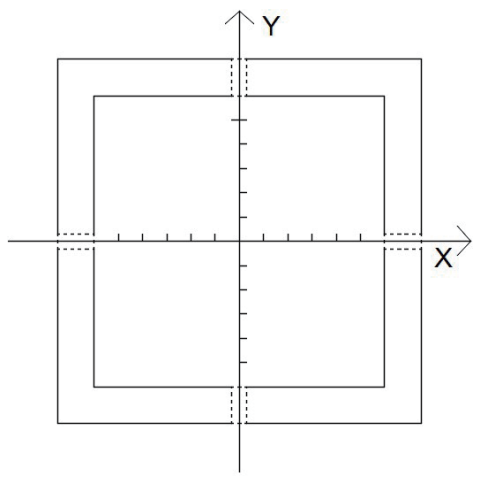

Fig. 3. Magnetic core coordinate system. 
$(12,0)$. The wire current was set to $10 \mathrm{~A}$. The magnetic induction intensity at the air gap was measured when the wire was located at different positions, and the relative error was obtained by comparing with the magnetic induction intensity at the central position. It can be seen from Fig. 4 that the farther the conductor is from the center, the greater the relative error.

Using the same core parameters, coordinate system, and current value, we simulate the single-core dual-air-gap structure. The air gap relative coordinates are Air gap $1(12,0)$ and Air gap $2(-12,0)$. Table 3 shows the two air gaps' magnetic induction intensities when the wire is in different positions. As can be seen from Table 3, when the wire is close to one of the air gaps, the magnetic induction intensities of the two air gaps will generate opposite changes.

Figure 1(b) shows the symmetrical dual-core four-air-gap structure. Assuming that the measured wire moves vertically upward, that is, close to Hall 1 on magnetic core $\mathrm{C} 1$ and away from Hall 4 on magnetic core $\mathrm{C}$, the magnetic induction intensity of the two air gaps on the magnetic core $\mathrm{C} 1$ will generate opposite changes. The corresponding output voltages $U_{1}$ and $U_{4}$ will also change accordingly, but $\left(U_{1}+U_{4}\right) / 2$ reduces the drift due to the vertical deviation of the conductor. Similarly, for core $\mathrm{C} 2$, since the two air gaps are symmetric with respect to the center of the core, when the conductor is moved horizontally, $\left(U_{2}+U_{3}\right) / 2$ can also reduce the amount of drift due to the horizontal deviation of the conductor position.

Although the measured wire deviates from the center of the core, the average output voltage of the proposed structure can reduce the error. Therefore, the error caused by the deviation of the measured wire from the center of the magnetic core is significantly reduced.

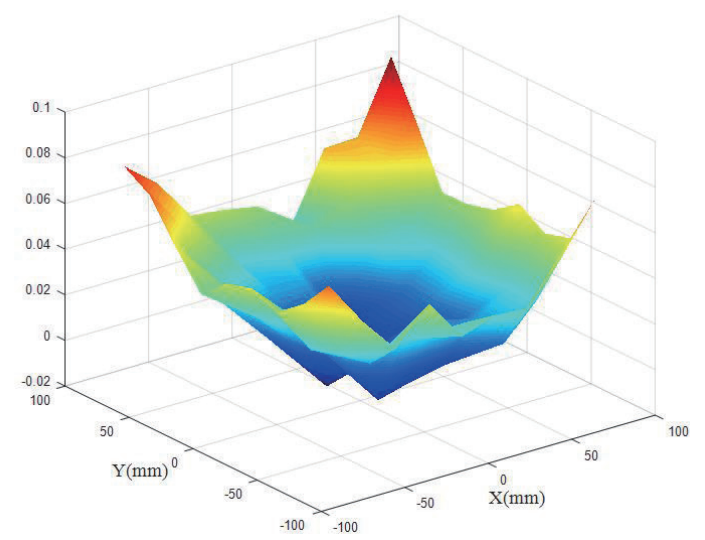

Fig. 4. (Color online). Relative error of air gap magnetic induction intensity in a single-core, single-air-gap structure when the conductor is at different locations.

Table 3

Magnetic induction intensities of the two air gaps when the wire is in different positions.

\begin{tabular}{lccccccccc}
\hline Wire coordinates & $(0,0)$ & $(-2,0)$ & $(2,0)$ & $(-6,0)$ & $(6,0)$ & $(-2,-2)$ & $(2,2)$ & $(-6,-6)$ & $(6,6)$ \\
\hline Air gap 1 (mT) & 2.447 & 2.349 & 2.567 & 2.143 & 2.864 & 2.372 & 2.593 & 2.321 & 2.825 \\
Air gap 2 (mT) & 2.447 & 2.576 & 2.342 & 2.875 & 2.135 & 2.601 & 2.365 & 2.857 & 2.323 \\
\hline
\end{tabular}




\section{Simulation Verification}

From the above analysis, the reason for the position error is that the measured conductor deviates from the center of the magnetic ring and the magnetic induction intensity at the air gap changes. To study the variation of the magnetic induction intensity in the air gap of the magnetic core with the deviation of the conductor position, a COMSOL finite element simulation was conducted.

The coordinate system shown in Fig. 3 was established for the magnetic core. The traditional single-core single-air-gap structure and the new structure take the center of the magnetic core as the origin of the coordinates, and relative coordinates are used to represent the different positions of the wire in the magnetic core. Figure 5 shows the finite element simulation model of the dual-core four-gap structure.

The new structure is simulated using COMSOL software to obtain the average magnetic induction intensity of the four air gaps and calculate the relative errors of the conductors at different positions. The relative errors of the new structure are compared with those of air gaps with the traditional structure for different currents $I_{P}$. The results for currents of 10,20,50, and $100 \mathrm{~A}$ are shown in Figs. 6(a)-6(d), respectively. The top three-dimensional layer in the figure is the simulation result obtained with the traditional structure, and the bottom three-dimensional layer is the simulation result obtained with the new structure.

It can also be seen in Fig. 6 that the new structure can reduce the measurement error significantly. When the currents are $10,20,50$, and $100 \mathrm{~A}$, the maximum relative position errors of the traditional structure are $0.918,0.862,0.831$, and $0.818 \%$, and the maximum relative position errors of the new structure are $0.468,0.485,0.474$, and $0.481 \%$, respectively.

In addition, because of the proportional relationship between the current and the magnetic induction intensity at the air gap, the simulation results for the relative errors at different currents are very close.

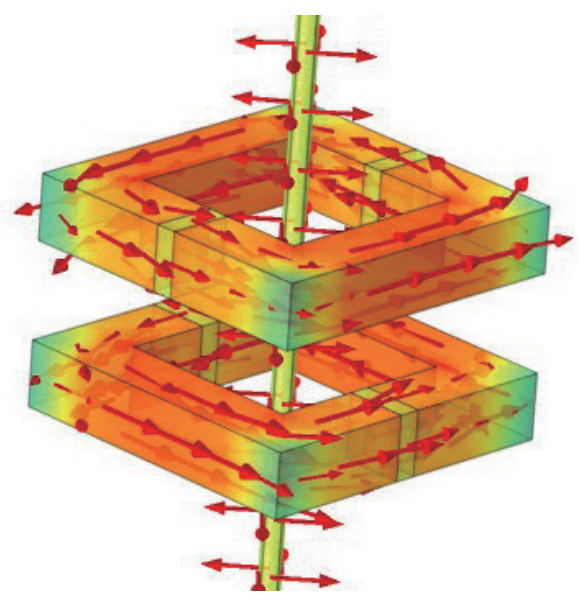

Fig. 5. (Color online) Finite element analysis model of dual-core four-air-gap structure. 


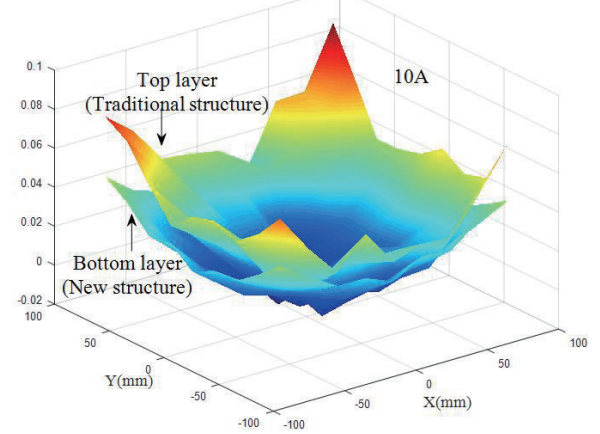

(a)

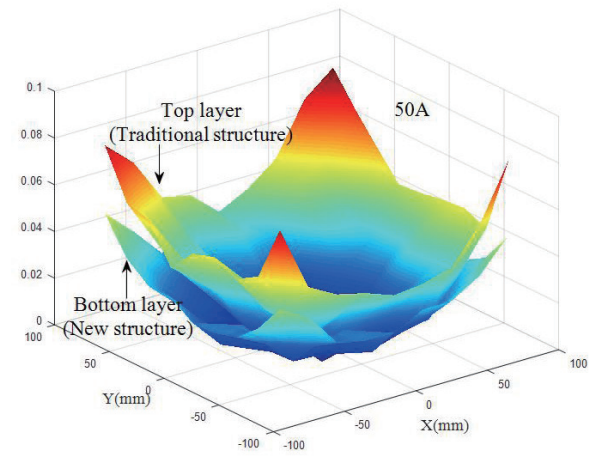

(c)

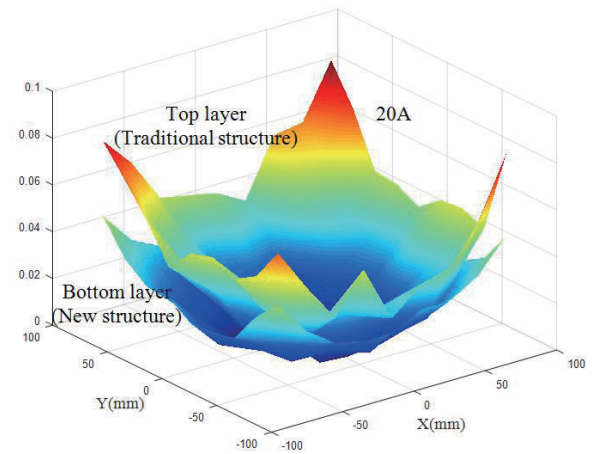

(b)

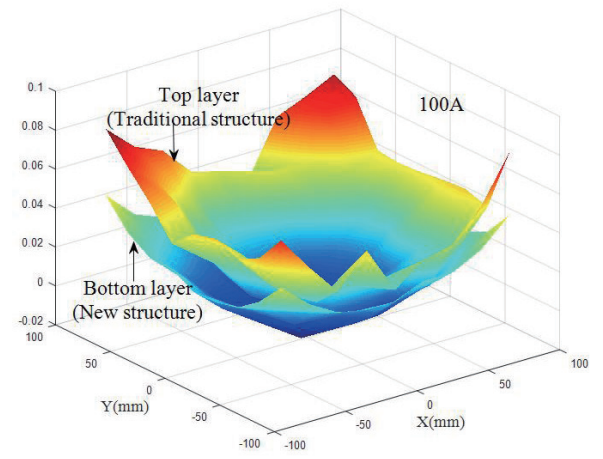

(d)

Fig. 6. (Color online) Relative errors of two structures obtained by simulation: (a) $I_{P}=10 \mathrm{~A}$, (b) $I_{P}=20 \mathrm{~A}$, (c) $I_{P}=$ $50 \mathrm{~A}$, (d) $I_{P}=100 \mathrm{~A}$.

\section{Experiment and Analysis}

\subsection{Test prototype}

The air gap design of the magnetic ring is very important for Hall current sensors. ${ }^{(11)}$ The air gap width should not be too large to avoid a weak magnet congregate effect and low sensitivity of the sensor. Moreover, if it is too small, the magnetic ring will be easily saturated, resulting in output distortion. Moreover, the air gap width must be greater than the thickness of the Hall chip package. The Hall chip is EG49A. After comprehensive consideration, the length of the air gap was set to $1.5 \mathrm{~mm}$. Figure 7(a) shows the dimensions of the magnetic core, for which ferrite was selected as the core material.

Figure 7(b) shows a sensor with a complementary symmetrical structure. Two identical dual-gap magnetic rings were placed cross-aligned and symmetrically with respect to the center of the core. To form two independent magnetic circuits, the middle of the two magnetic rings was isolated by printed circuit board (PCB), and the two magnetic rings were fixed by the corresponding skeleton. To ensure that the voltages of the four Hall chips increased with the current in the fixed direction of the measured conductor, two Hall chips on the same core were placed at the core air gap in reverse orientations. The prototype built to verify the performance of the proposed circuit is shown in Fig. 7(c). 


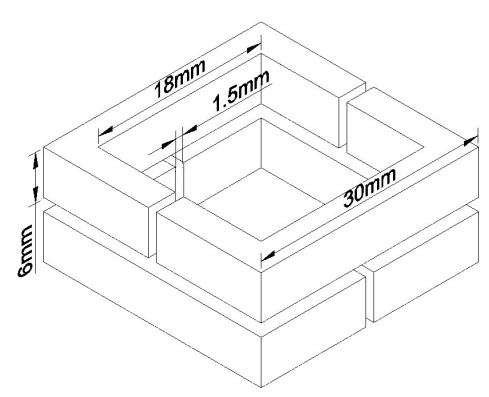

(a)

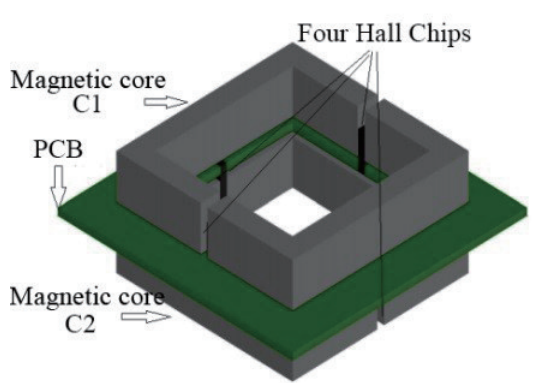

(b)

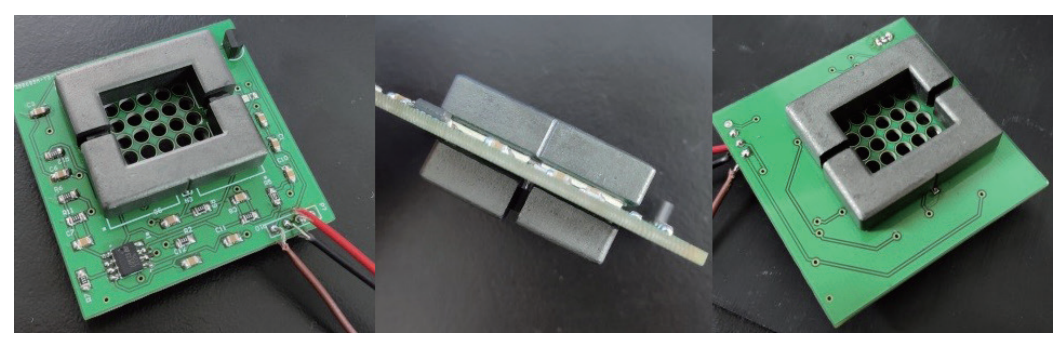

(c)

Fig. 7. (Color online) (a) Dimensions of the magnetic core. (b) Sensor with complementary symmetrical structure. (c) Sensor prototype.

\subsection{Experimental results}

Considering the diameter of the wire and other practical operations, we set a total of 25 position coordinates of the wire during the experiment, evenly distributed in the magnetic ring. The relative errors of the output voltage were calculated when the conductor was located at these different positions. Figures 8 (a) -8 (c) show the comparative experimental results of the traditional single-core single-air-gap structure and the new structure when the current was 10 , 20 , and $50 \mathrm{~A}$, respectively.

The top three-dimensional layer in the figure is the experimental results obtained with the traditional structure, and the bottom three-dimensional layer is the experimental results obtained with the new structure. According to the experimental data, when the currents were 10,20 , and $50 \mathrm{~A}$, the maximum relative position errors of the traditional structure were 0.912 , 0.988 , and $0.983 \%$ and the maximum relative position errors of the new structure were $0.566,0.550$, and $0.542 \%$, respectively. It can be seen from Fig. 8 that the maximum relative errors occurred near the vertices farthest from the center, which is also consistent with the simulation results. Thus, the new structure plays an important role in reducing the error.

In addition, the tested wire was placed at the center position of the two structures and the current was varied from -50 to $50 \mathrm{~A}$. The fitting straight lines of the two structures were compared, as shown in Fig. 9. Different air gaps and magnetic cores have different fitting results. According to the fitting straight lines of the two structures, the linearities were 


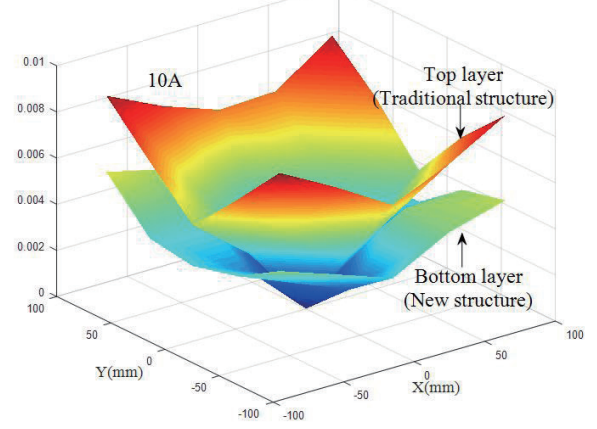

(a)

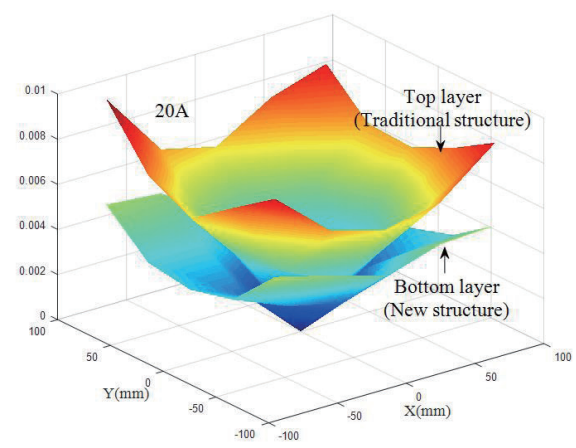

(b)

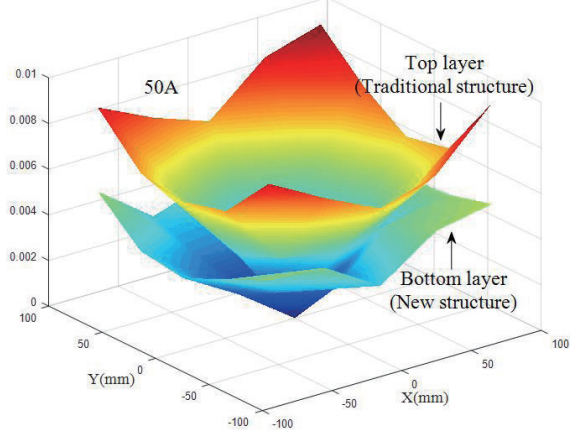

(c)

Fig. 8. (Color online) Relative errors of two structures obtained experimentally: (a) $I_{P}=10 \mathrm{~A}$, (b) $I_{P}=20 \mathrm{~A}$, (c) $I_{P}=50 \mathrm{~A}$.

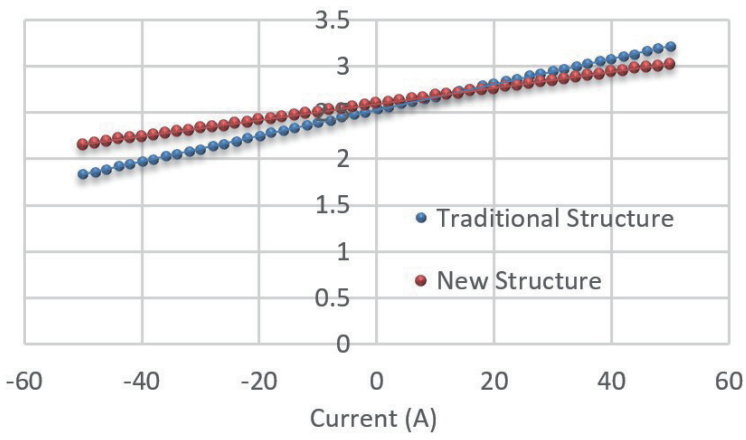

Fig. 9. (Color online) Fitting straight lines of two structures.

0.892 and $0.890 \%$. The linear error under the new structure is within $1 \%$, so it can meet the measurement requirements.

\section{Conclusions}

According to the results of simulation and theoretical and experimental analyses, the error of an open-loop Hall current sensor with the traditional single-air-gap structure cannot be 
ignored when the measured conductor deviates from the center position. The position variation of the measured conductor will affect the magnetic induction intensity in the air gap. Also, the larger the distance between the conductor and the center of the core, the greater the error. This error limits the accuracy of existing open-loop Hall current sensors. The improved dual-core four-air-gap complementary symmetrical core structure proposed in this paper was verified by simulation and experiment. The simulation and experiment results show that the proposed structure can effectively reduce the error caused by the position of the measured conductor deviating from the center of the magnetic ring, thus improving the measurement accuracy of open-loop Hall current sensors.

\section{References}

1 P. Ripka, P. Kejik, P. Kaspar, and K. Draxler: Instrum. Meas. Technol. Conf. 2 (1996) 1479. https://ieeexplore. ieee.org/document/507616

2 S. Ziegler, R. C. Woodward, H. H. Iu, and L. J. Borle: Sens. J. 9 (2009) 354. https://ieeexplore.ieee.org/ document/4797906/

3 A. P. Nurmansah and S. Hidayat: Int. Conf. High Voltage Engineering and Power Systems (IEEE, 2017) 493497.

4 R. Weiss, R. Makuch, A. Itzke, and R. Weigel: IRE Trans. Ind. Electron. 64 (2017) 4903. https://ieeexplore. ieee.org/document/7864359

5 R. Weiss, A. Itzke, and R. Weigel, 2017 IEEE 2nd Int. Conf. DC Microgrids (IEEE, 2017) 166-170.

6 R. Weiss, A. Itzke, J. Reitenspieß, I. Hoffmann, and R. Weigel: Sens. J. 19 (2019) 2517. https://ieeexplore.ieee. org/document $/ 8581469$

7 D. Rienzo, R. Bazzocchi, and A. Manara: Trans. Instrum. Meas. 50 (2001) 1093. https://ieeexplore.ieee.org/ document/963165/

8 H. Yu, Z. Qian, H. Liu, and J. Qu: Magn. Sens. 18 (2018) 578. https://www.mdpi.com/1424-8220/18/2/578

9 A. Itzke, R. Weiss, and R. Weigel: Trans. Ind. Electron. 66 (2019) 580. https://ieeexplore.ieee.org/ document $/ 8336943$

10 R. S. Popovic, Z. Randjelovic, and D. Manic: Sens. Actuators, A 91 (2001) 46. https://www.sciencedirect.com/ science/article/abs/pii/S0924424701004782

11 C. Xu, J. Liu, Q. Zhang, C. Xu, and Y. Yang: 2015 IEEE Int. Workshop on Applied Measurements for Power Systems (IEEE, 2015) 19-24.

12 A. Ajbl, M. Pastre, and M. Kayal: SENSORS, 2012 IEEE (2012) 1. https://ieeexplore.ieee.org/ document/6411298/

13 X. Cheng, Z. Zhang, and F. Li: J. Comput. Theor. Nanosci. 11 (2014) 1121. https://www.ingentaconnect.com/ content/asp/jctn/2014/00000011/00000004/art00027

14 F. Li, X. G. Cheng, S. Liu, and X. B. Luo: 2012 14th Int. Conf. Electronic Materials and Packaging (IEEE, 2012) $1-5$.

15 X. G. Cheng, F. A. Li, and Z. Y. Zhang: Sci. China: Technol. Sci. 57 (2014) 1877. http://www.cnki.com.cn/ article/cjfdtotal-jexg201409023.htm

16 Q. Zhang, J. Liu, and Y. Yang: Proc. PCIM Europe 2015-International Exhibition and Conference for Power Electronics, Intelligent Motion, Renewable Energy and Energy Management 19 (IEEE, 2015) 1-8.

17 R. Li, H. Qiao, and C. Zhang: Appl. Mech. Mater. 494 (2014) 1462. https://www.scientific.net/AMM.494495.1462 
